\title{
DIATOMEAS HOLOCENAS DEL DELTA DEL RÍO COLORADO, ARGENTINA. UNA APROXIMACIÓN PALEOAMBIENTAL A PARTIR DEL ESTUDIO DE “FRAGILARIOIDES PEQUEÑAS”
}

\author{
ROCÍO FAYÓ (D, MARCELA ALCIRAESPINOSA (iD \& FEDERICO IGNACIO ISLA (D) \\ Instituto de Geología de Costas y del Cuaternario, (IGCyC), Universidad Nacional de Mar del Plata (UNMdP)/ Comisión \\ de Investigaciones Científicas de la Provincia de Buenos Aires (CIC). Instituto de Investigaciones Marinas y Costeras \\ (IIMyC), UNMdP/Consejo Nacional de Investigaciones Científicas y Técnicas (CONICET), \\ Rodríguez Peña 4046, Mar del Plata (7600), Buenos Aires, Argentina. \\ rociofayo@gmail.com,maespin@mdp.edu.ar,fisla9@gmail.com
}

\begin{abstract}
Holocene diatoms of the Colorado River Delta, Argentina. A paleoenvironmental approach from the study of "small fragilarioids". Diatom assemblages of a sedimentary succession in the deltaic plain of the Colorado River (Argentina) were studied in order to reconstruct the paleoenvironmental conditions of the delta in response to Holocene sea-level changes. A $191 \mathrm{~cm}$ depth core was extracted by vibracoring from Las Isletas (LI), located $22 \mathrm{~km}$ from the coast (3943'5.7'S, 62 $\left.20^{\prime} 49.7^{\prime \prime} \mathrm{W}\right)$. A cluster analysis - CONISS of 35 subsamples defined three diatom zones (DZ). The sedimentary succession is dominated by small fragilarioids, being Punctastriata glubokoensis Williams, Chudaev \& Gololobova the most abundant species; the accompanying flora included fresh-brackish and allochthonous marine-coastal taxa. At the base, subzone DZIA $\left(191-163 \mathrm{~cm}, 7,315 \pm 64{ }^{14} \mathrm{C}\right.$ yr BP) included sandy lithological units and presented abundant marine-coastal species. The overlying subzone DZIB $(163-96 \mathrm{~cm})$ has low abundance of allochthonous marine species. The uppermost zone, DZII (96 cm from the top) integrate the silt-sandy lithological units, and it was deposited ca. $2,218 \pm 29{ }^{14} \mathrm{C}$ yr BP. DZII lacked marine species. The LI sequence represents the infilling of a river-estuarine channel with tidal influence $c a$. $7,300{ }^{14} \mathrm{C}$ yr $\mathrm{BP}$ that gradually evolved into a fresh-brackish river channel, similar to the current river, $c a .2,200{ }^{14} \mathrm{C}$ yr BP. During the Middle Holocene transgression, the greatest marine influence would have transported coastal marine species upstream to the LI site; the increase of fluvial influence associated with the progradation of the delta lobe would have favored the ubiquity and persistence of the small fragilarioids in the fluvial-delta assemblages of the Colorado River during the last $c a .7,000{ }^{14} \mathrm{C}$ yr BP.
\end{abstract}

Keywords: Fragilariaceae, delta, sea level, Holocene, Patagonia.

RESUMO - As associações de diatomáceas, de uma sucessão sedimentar da planície deltaica do Rio Colorado (Argentina), foram estudadas com o objetivo de reconstruir as condições paleoambientais do delta, em resposta às flutuações do nível do mar no Holoceno. Um testemunho de $191 \mathrm{~cm}$ de profundidade foi extraído usando um vibracoring no sitio Las Isletas (LI), localizado a $22 \mathrm{~km}$ da costa atual (3943'5,7'S, $62^{\circ} 20^{\prime} 49,7$,O). A análise diatomológica de 35 sub-amostras permitiu definir três zonas de diatomáceas (DZ) por meio de uma análise de agrupamento - CONISS. O testemunho foi dominado por pequenos fragilarioides, sendo Punctastriata glubokoensis Williams, Chudaev \& Gololobova a espécie mais abundante. A flora acompanhante apresentou espécies de água salobra-duciaquícolas e táxons marinho-costeiros alóctones. A subzona basal DZIA (191-163 cm, $7.315 \pm 64$ anos ${ }^{14} \mathrm{C}$ AP) incluiu unidades litológicas arenosas e apresentou abundantes diatomáceas marinhas costeiras alóctone. A subzona sobrejacente (DZIB: 163-96 cm) exibiu poucos táxons marinhos. A zona DZII (96 $\mathrm{cm}$ no topo) integrou unidades litológicas de areia-siltosa, formadas $c a .2 .218 \pm 29$ anos ${ }^{14} \mathrm{C}$ AP. DZII e não apresentou espécies marinhas costeiras. A sequência LI representa o assoreamento de um canal fluvial-estuarino com influência de marés $c a$. 7.300 anos ${ }^{14} \mathrm{C}$ AP que evoluiu gradualmente para um canal fluvial de águas salobra-dulciaquícolas, semelhante ao rio atual $c a$. 2.200 anos ${ }^{14} \mathrm{C}$ AP. Durante a transgressão do Meso-holoceno, o aumento da influência marinha teria transportado espécies costeiras-marinhas a montante do sítio LI. O aumento da influência fluvial associada à progradação do lobo deltaico teria favorecido a onipresença e a persistência de pequenos fragilarioides nas associações fluvio-deltaica do Rio Colorado durante os últimos ca. 7.000 anos ${ }^{14} \mathrm{C} \mathrm{AP}$.

Palavras-chave: Fragilariaceae, delta, nível do mar, Holoceno, Patagônia.

\section{INTRODUCCIÓN}

Las diatomeas pennadas arrafídeas de la familia Fragilariaceae son importantes componentes de los ensambles de microalgas planctónicas y bentónicas de ambientes continentales salobre-dulceacuícolas y costero-marinos (Witkowski et al., 2000; Morales, 2005; Metzeltin \& LangeBertalot, 2007; Li et al., 2016). Las diferentes especies han sido descriptas tradicionalmente por la morfometría del frústulo silíceo utilizando microscopía de luz (LM); sin 
embargo, los representantes de menor tamaño, generalizados como fragilarioides pequeñas, presentan valvas menores de 20 $\mu \mathrm{m}$ y exhiben morfologías similares, lo que hace virtualmente imposible discernir sus caracteres diagnósticos de detalle (Morales, 2001; Paull et al., 2008). Esta limitación en el análisis específico ha generado información fragmentaria respecto a su abundancia, distribución y en consecuencia una subestimación de la diversidad de los taxones pennados arrafídeos pequeños (Li et al., 2019).

Si bien los representantes de la familia Fragilariaceae son muy abundantes y han sido estudiados exhaustivamente durante las últimas dos décadas, la taxonomía de las especies y géneros que la componen es aún discutida (Cox, 2012; Guerrero et al., 2019). La clasificación de los taxones pertenecientes a este grupo ha cambiado sustancialmente debido a la incorporación de nuevos criterios diagnósticos basados en el estudio de la ultraestructura del frústulo con microscopía electrónica de barrido (MEB) y las evidencias moleculares (Medlin et al., 2012; Theriot et al., 2015; García et al., 2017; Li et al., 2019). Las revisiones del material tipo con mayor resolución microscópica y el incremento en número de las investigaciones, han propiciado la reinterpretación y redistribución de la diversidad morfológica inicialmente adjudicada al género Fragilaria Williams \& Round (1987) sensu lato hacia un número creciente de nuevos géneros y especies (Williams \& Round, 1987; Flower et al., 1996; Round et al., 1990; Cejudo-Figueiras et al., 2011). De forma consistente, los análisis filogenéticos de Fragilariaceae indican un origen parafilético (Medlin et al., 2012; Theriot et al., 2015). Desde la perspectiva ecológica las fragilarioides pequeñas son ubicuas en el ambiente y por lo general coexisten en los ensambles; en este sentido, un diagnóstico taxonómico inespecífico también interfiere en la determinación de sus afinidades ecológicas (Schmidt et al., 2004; Vélez-Agudelo et al., 2017).

Los obstáculos mencionados constituyen una restricción metodológica para la utilización de estos microfósiles en reconstrucciones paleoambientales, donde las especies constituyen los proxy-data, y la precisión taxonómica es fundamental para extrapolar la información autoecológica a la interpretación (Díaz Pardo et al., 2008; Hermany et al., 2013; Morales et al., 2014). En el registro fósil, los estudios que aplican estos avances en la comprensión de la diversidad de la familia Fragilariaceae han sido escasos. Por lo tanto, la diversidad de las diatomeas pennadas arrafídeas pequeñas en escalas temporales amplias se encuentra subrepresentada. En este sentido, es necesario incrementar los análisis de sus taxones fósiles mediante el uso combinado de LM y MEB de modo para reconocer la diversidad morfológica de los taxones y su ubicuidad en los ambientes del pasado.

Las fragilarioides pequeñas son abundantes en los ambientes ácueos actuales de Argentina, tanto naturales como artificiales. Han sido ampliamente identificadas en muestras de sedimento superficial, perifiton y en el plancton de lagos, ríos, arroyos, humedales, estanques y embalses (Maidana \& Díaz-Villanueva, 2001; Espinosa \& Isla, 2015; VélezAgudelo et al., 2017). También se han registrado en secuencias sedimentarias de estuarios y ambientes continentales de Buenos Aires y Patagonia (Espinosa, 2008; Escandell et al., 2009; Hassan et al., 2009, 2011; Espinosa et al., 2012; Fayó \& Espinosa, 2014). Sin embargo, sólo los trabajos más recientes abordan la identificación taxonómica de las diatomeas fósiles con los nuevos criterios de clasificación y el uso combinado de LM y MEB (e.g. García \& Maidana, 2015; García et al., 2017; Fayó et al., 2018, Grana et al., 2018).

La Cuenca del río Colorado, ubicada en la región norte de la Patagonia, presenta exiguos registros diatomológicos respecto a otras regiones de Argentina. En su desembocadura forma uno de los dos sistemas deltaicos del país. Biasotti et al. (2013, 2014) y Galea et al. (2014) realizaron un análisis estacional de la composición de diatomeas fitoplanctónicas en su sección media; Vouilloud \& Leonardi (2001) estudiaron la composición de los ensambles de los canales de drenaje de su sección inferior, mientras que Garibotti et al. (2011) realizaron análisis diatomológico estacional del fitoplancton del estuario de Bahía Anegada, al sur de la desembocadura del río Colorado. Sin embargo, estos antecedentes no hacen referencia a la presencia de diatomeas arrafídeas pennadas pequeñas en los ensambles. Vélez-Agudelo et al. (2017) estudiaron la composición y distribución espacial de la flora diatomológica moderna contendida en sedimentos superficiales de la cuenca media e inferior del río Colorado y establecieron el primer registro en Argentina de dos especies del género Punctastriata: $P$. glubokoensis Williams, Chudaev \& Gololobova y P. lancettula Schumann Hamilton \& Siver, e identificaron a Staurosira venter (Ehrenberg) Cleve \& Möller como la especie dominante de los ensambles fluviales salobre-dulceacuícolas. Respecto al registro fósil, las sucesiones sedimentarias holocenas de la llanura deltaica del río Colorado se encuentran prácticamente inexploradas desde una perspectiva diatomológica. Hasta el momento Fayó et al. (2018) realizaron la reconstrucción paleoambiental de un testigo sedimentario extraído de su llanura deltaica a una distancia de $37 \mathrm{~km}$ de la línea de costa actual. En tal oportunidad se reconocieron pequeñas diatomeas arrafídeas como componentes abundantes de los ensambles fósiles de los últimos $4.132 \pm 35$ años ${ }^{14} \mathrm{C}$ AP $(4510-4714$ años cal. AP); y su incremento durante los últimos 150 años AP fue asociado al establecimiento de las condiciones fluviales análogas a las actuales. Ese antecedente remarca el primer registro holoceno de Punctastriata glubokoensis y Pseudostaurosiropsis geocollegarum (Witkowski \& LangeBertalot) Morales para Argentina.

El objetivo de esta contribución es realizar la reconstrucción paleoambiental basada en diatomeas de una sucesión sedimentaria de la llanura deltaica del río Colorado Argentina, e incrementar el conocimiento de la diversidad de la flora diatomológica, con especial énfasis en las diatomeas pennadas arrafídeas pequeñas conocidas como "fragilarioides pequeñas".

\section{ÁREA DE ESTUDIO}

El río Colorado es un curso alóctono que nace de la confluencia del río Barrancas y el río Grande en la zona andina media de Argentina y discurre $923 \mathrm{~km}$ en sentido NO-SE a 
través de las mesetas patagónicas y la llanura pampeana, constituyendo un límite natural entre ambas regiones. $\mathrm{Su}$ cuenca inferior se localiza al sur de la Provincia de Buenos Aires, donde el río forma un lóbulo deltaico operativo que prograda en la costa abierta del Océano Atlántico (Spalletti \& Isla, 2003; Figura 1). El delta se extiende desde la Península Verde $\left(39^{\circ} 20^{\prime} \mathrm{S}, 62^{\circ} 4^{\prime} \mathrm{O}\right)$ hasta la Isla Otero $\left(39^{\circ} 55^{\prime} \mathrm{S}\right.$, $\left.62^{\circ} 08^{\prime} \mathrm{O}\right)$. Se trata de un delta mixto lobulado destructivo con retrabajamiento por el oleaje, que desemboca en costas mesomareales con mareas semidiurnas a mixtas (Spalletti \& Isla, 2003). La llanura deltaica no posee actividad tectónica significativa y actualmente representa un remanente de un sistema deltaico antiguo de mayor magnitud (Melo et al., 2013). La misma se compone de lóbulos deltaicos pleistocenos, numerosos cursos afuncionales que han tenido un régimen distributario en el pasado y lagunas en medialuna colmatadas (Weiler, 1980; Spalletti \& Isla, 2003). Además, contiene antiguas líneas de costa con evidencia malacológica holocena que nos permiten estimar una tasa de progradación de $5 \mathrm{~m} /$ año. Entre los canales distributarios meandrosos activos o parcialmente activos se destacan de norte a sur, el río Colorado Nuevo, el Colorado y el Colorado Viejo (Figura 1). El río Colorado es el cauce troncal que discurre por el centro del valle, mientras que los otros han sido intermitentes en tiempos históricos, reactivándose durante los pulsos de inundación o precipitaciones copiosas (Charó et al., 2015). La formación del delta es favorecida por la baja pendiente de la cuenca y un importante aporte sedimentario fluvial, siendo el caudal sólido medio anual de 569.000 toneladas de sedimento (Blasi, 1986).

Debido a su gran extensión, el río Colorado recorre un gradiente de precipitación que se incrementa hacia el este, atravesando condiciones áridas a semiáridas-templadas, con un promedio anual de precipitación de $250 \mathrm{~mm}, 320 \mathrm{~mm}$, $384 \mathrm{~mm}$ en la cuenca alta, media y baja respectivamente (COIRCO, 2018). El río posee un régimen pluvio-nival con caudales anuales promedio de $140 \mathrm{~m}^{3} \mathrm{~s}^{-1}$ y alcanza valores máximos estacionales durante primavera-verano $\left(818 \mathrm{~m}^{3} \mathrm{~s}^{-1}\right.$; González Uriarte, 1984). Presenta aguas levemente alcalinas durante todo el año ( $\mathrm{pH}=8,35 \pm 0,58)$, mientras que otras variables ambientales muestran un patrón estacional (Biasotti et al., 2013; Vélez-Agudelo et al., 2017). El río Colorado posee un mayor rango de salinidad respecto a otros ríos patagónicos (Espinosa \& Isla, 2015), con menor tenor salino durante en los meses de primavera-verano $(0,56 \pm 0,06) \mathrm{y}$ mayor en invierno $(0,84 \pm 0,13)$ cuando el caudal es mínimo (Vélez-Agudelo et al., 2017).

\section{MATERIAL Y MÉTODOS}

El testigo fue extraído mediante técnicas de vibracoring de la llanura deltaica del río Colorado, en el sitio denominado Las Isletas (LI: $39^{\circ} 43^{\prime} 5,7^{\prime \prime}$ S, $62^{\circ} 20^{\prime} 49,7^{\prime \prime}$ ) ), ubicado a 22 $\mathrm{km}$ de la línea de costa actual. En el laboratorio fue abierto longitudinalmente, se realizó la descripción litológica según el color de los sedimentos, tamaño de grano y presencia de estructuras sedimentarias y se submuestró cada $2 \mathrm{~cm}$. Según los cambios sedimentarios, se seleccionaron 35 submuestras para el análisis diatomológico. Con el fin de concentrar las diatomeas y retirar la arena fina, se realizó una decantación diferencial en un intervalo de tiempo controlado. La mitad de la fracción submuestreada $\left(38,5 \mathrm{~cm}^{3}\right.$ de sedimento) fue disgregada y homogeneizada con agua destilada en una probeta de 11; luego se decantó durante 20 segundos y se recuperó el sobrenadante con la fracción pelítica y las diatomeas (Sterrenburg, 2006) y el precipitado con la fraccion psammitica fue descartado. La muestra concentrada se reservó en frascos de $50 \mathrm{ml}$. Posteriormente una alícuota ( $3 \mathrm{ml}$ ) fue tratada a $80^{\circ} \mathrm{C}$ con $\mathrm{H}_{2} \mathrm{O}_{2}$ y $\mathrm{HCl}$ para remover la materia orgánica y los carbonatos respectivamente (Battarbee et al., 2001). Se realizaron preparados definitivos con Naphrax ${ }^{\circledR}(R I=1.75)$ para la identificación taxonómica y se contabilizaron al menos 300 valvas por muestra (1000X) con un microscopio óptico Zeiss Axiostar plus (Crosta \& Koç,

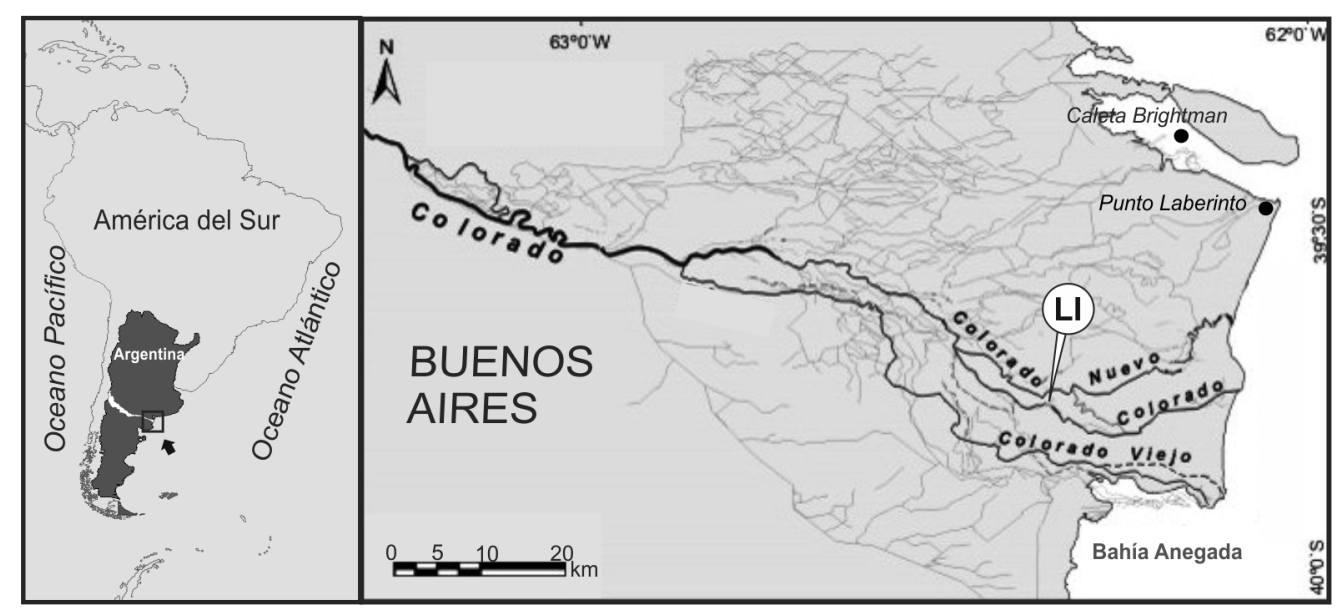

Figura 1. Mapa de ubicación de la Cuenca del río Colorado (Argentina). El sitio de estudio Las Isletas (LI) se ubica en su llanura deltaica al SE de la Provincia de Buenos Aires.

Figure 1. Location map of the Colorado River Basin (Argentina). The study site 2Las Isletas (LI)" is located in its deltaic plain to the SE of the Province of Buenos Aires. 
2007). Como recurso diagnóstico de mayor resolución, se utilizó el Microscopio Electrónico de Barrido del Laboratorio de Microscopía Electrónica de la Universidad Nacional de Mar del Plata (Jeol-JSM 6460LV) y el MEB de alta resolución de la Universidad Nacional de Buenos Aires (CMA-UBA, Zeiss-SUPRA40).

La identificación de las especies fue basada en literatura taxonómica específica tradicional y la sinonimia fue establecida mediante Algaebase (Guiry \& Guiry, 2020) y DiatomBase (Kociolek et al., 2018). La cronología de la sucesión sedimentaria fue establecida en base a dos dataciones radiocarbónicas AMS $\left({ }^{14} \mathrm{C}\right.$ años AP) en Direct AMS Laboratory (Washington, EEUU), mientras que las edades calibradas ( $\mathrm{cal}$ años AP) fueron calculadas a través del programa CALIB 5.0.1, (Stuiver et al., 2005) utilizando la curva de calibración para el hemisferio sur (SHCal04, McCormac et al., 2004).

\section{Análisis de datos}

Los conteos fueron expresados como frecuencias relativas en porcentajes y sólo aquellos taxones con más de $5 \%$ en al menos una muestra, fueron incluidos en la interpretación. Las diatomeas fueron clasificadas en relación con sus tolerancias a la salinidad y formas de vida, siguiendo la clasificación ecológica de Vos \& de Wolf (1993), Denys (1991/1992) y estudios ecológicos regionales complementarios (e.g. Echazú, 2012; Vélez-Agudelo et al., 2017). Las zonas diatomológicas fueron definidas utilizando el análisis de agrupamiento jerárquico restringido (constrained hierarchical clustering - CONISS) y la distancia de Bray Curtis. Con el fin de estabilizar la varianza de los datos biológicos, las frecuencias relativas de las especies fueron transformadas a su raíz cuadrada (Bigler \& Hall, 2002), respetando el orden estratigráfico de las muestras. El número de zonas diatomológicas estadísticamente significativas fue establecido con el modelo Broken-stick (Bennett, 1996). Los análisis estadísticos y gráficos fueron realizados con el software $\mathrm{R}$ versión 3.2.2 (R Core Team, 2015), utilizando los paquetes "Rioja" versión 0.9-5 (Juggins, 2015) y el "Vegan" versión 2.3-0 (Oksanen et al., 2015).

\section{RESULTADOS}

\section{Cronología y análisis sedimentológico}

La sucesión sedimentaria LI presentó $191 \mathrm{~cm}$ de potencia y abarcó los últimos $c a$. 7.300 años AP. Las edades convencionales (años ${ }^{14} \mathrm{CAP}$ ) y las edades calibradas (años cal $\pm 2 \delta$ ) se determinaron a partir del análisis de materia orgánica en sedimento, siendo la edad basal de la sucesión sedimentaria (187-189 cm) de 7.315 \pm 64 años ${ }^{14} \mathrm{C} \mathrm{AP}(8.001-8.177$ años cal AP). Una segunda datación realizada a $37-39 \mathrm{~cm}$, permitió determinar una edad de $2.218 \pm 29$ años ${ }^{14} \mathrm{C}$ AP $(2.140-2.309$ años cal AP).

Se identificaron cinco unidades litológicas diferenciables según su granulometría y estructuras sedimentarias (U1U5; Figura 2). Toda la secuencia presenta sedimentos con abundantes trizas vítreas y pumicita. La sección comprendida por las unidades U1-U4 se extiende desde 191 a $42 \mathrm{~cm}$ de profundidad y está conformada por arena fina de color castaño. Particularmente, la U1 basal $(191-130 \mathrm{~cm})$ presenta láminas horizontales de color castaño claro de diferente espesor (3-8 $\mathrm{mm}$ ) donde dominan clastos redondeados de tamaño arena media de pumicita. Las láminas de mayor espesor se encuentran en la base $(192-180 \mathrm{~cm})$ y luego hacia el tope de la unidad se hacen más finas. La unidad litológica suprayacente U2 (130-108 cm) está constituida por arena de color castaño, masiva y uniforme. Sobre ella, la U3 (80-108 $\mathrm{cm})$ posee estructuras convolutas y láminas horizontales de minerales oscuros y opacos. La unidad litológica U4 (80-42 $\mathrm{cm})$, se compone de arena castaña masiva uniforme sin laminaciones. Hacia el tope de la sucesión sedimentaria, los últimos $42 \mathrm{~cm}$ constituyen la quinta unidad litológica (U5) compuesta por granulometrías areno-limosas. En la sección basal de U5 se identifica mayor presencia de fangos, materia orgánica, y bioturbaciones de raíces; mientras que, hacia el tope decrece la abundancia de fangos y aumenta la de arena fina y muy fina.

\section{Análisis diatomológico}

El análisis diatomológico de las 35 submuestras permitió reconocer 179 taxones (incluyendo especies y variedades). La mayoría de estos frústulos presentaron un estado de preservación adecuado para la identificación taxonómica y no se observaron zonas estériles. El análisis específico de los ensambles permitió identificar 12 especies de diatomeas con una abundancia mayor al $5 \%$ en al menos una muestra. Sus proporciones fueron graficadas en un diagrama de frecuencias relativas (Figura 2). Se identificaron tres zonas diatomológicas estadísticamente significativas mediante el análisis de agrupamiento (CONISS) y el modelo de Brokenstick. Las muestras se agruparon según la presencia o ausencia de flora marino-costera en dos zonas principales (DZI y DZII respectivamente), cuyo límite se encuentra a los $96 \mathrm{~cm}$ de profundidad.

La zona diatomológica inferior de la sucesión sedimentaria LI (DZI: 191-96 cm) integró las unidades litológicas arenosas U1, U2 y la sección basal de U3. Está caracterizada por el dominio de Punctastriata glubokoensis con abundancias de 30-35\%. DZI presenta de forma característica una flora acompanante mixta con taxones marino-costeros (abundancias $<20 \%$ ) y salobres/dulceacuícolas (abundancias $<40 \%$ ). En función de las variaciones en la proporción de los mismos se delimitaron las subzonas DZIA y DZIB.

La subzona DZIA basal $(191-163 \mathrm{~cm})$ fue datada en $7.315 \pm 64$ años ${ }^{14} \mathrm{C}$ AP $(8.001-8.177$ años cal AP), y si bien dominaron los taxones salobres-dulceacuícolas, esta subzona presenta la mayor proporción de especies marinocosteras halladas en la secuencia. Estas últimas estuvieron representadas por Paralia sulcata (Ehrenberg) Cleve, 1873 (12\%) y Cymatosira belgica Grunow, 1881 (8\%), ambas con un importante grado de fragmentación valvar (Figura 3). Las especies del grupo de las fragilarioides pequeñas 


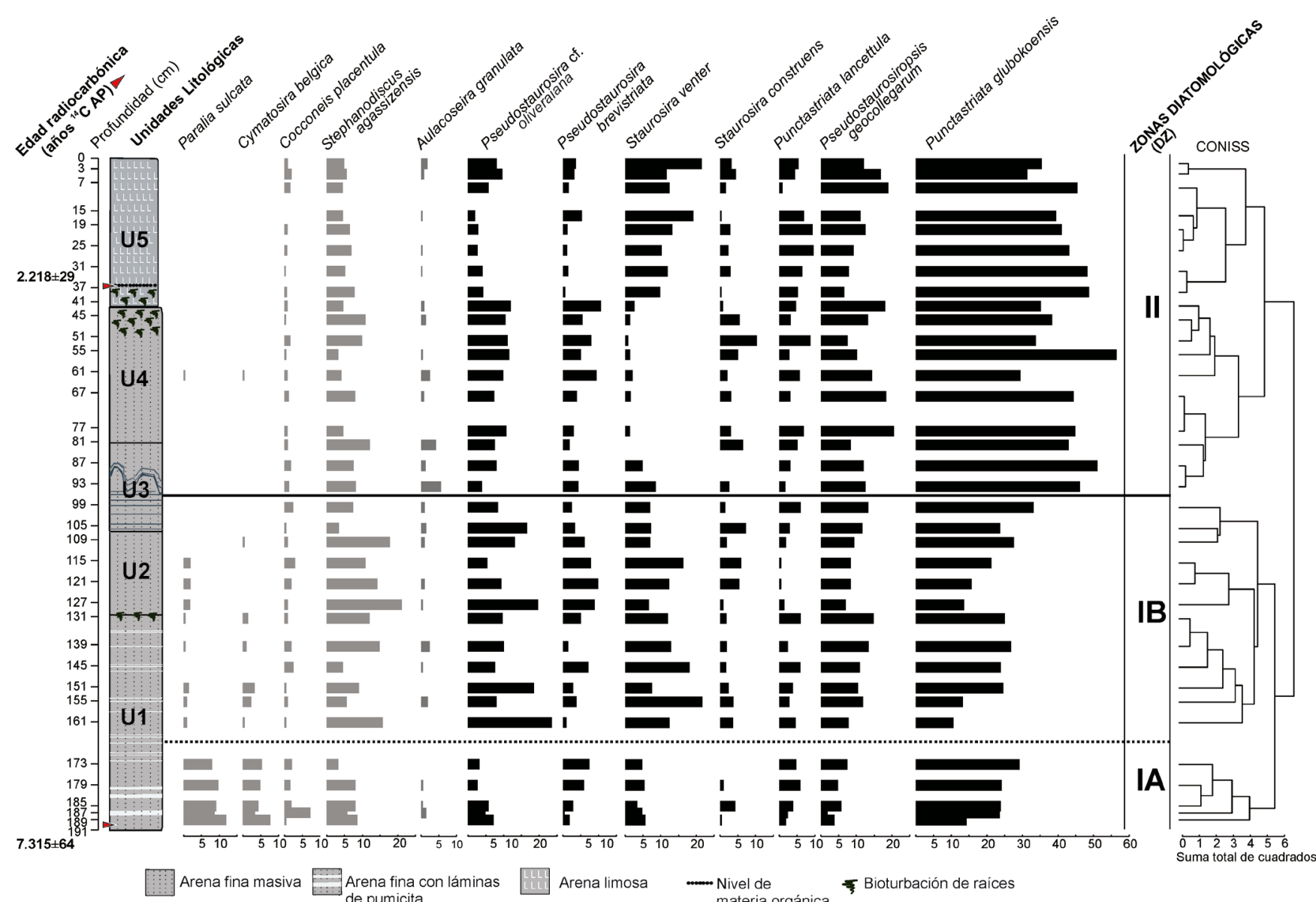

Figura 2. Perfil litológico de la sucesión sedimentaria LI, diagrama de frecuencias relativas de especies de diatomeas y análisis de agrupamiento CONISS. Las diatomeas pequeñas de la familia Fragilariaceae se muestran en negro.

Figure 2. Lithological profile of LI sedimentary succession, relative frequencies diagram of diatom species and CONISS cluster analysis. Small diatoms of the family Fragilariaceae are shown in black.

acompañantes, presentaron abundancias de 5 a $10 \%$ cada una, mientras que las diatomeas céntricas planctónicas, Stephanodiscus agassizensis Håkansson \& Kling, 1989 y Aulacoseira granulata (Hustedt) Simonsen, 1979, 8 y 2\%, respectivamente. La subzona suprayacente DZIB (163-96 $\mathrm{cm}$ ) fue delimitada en función de una disminución en las especies marinas $(<5 \%)$ y el incremento en la abundancia de especies fragilarioides y los taxones planctónicos. La zona diatomológica DZII (96 cm al tope) integró las unidades litológicas U3-U5 en las que el porcentaje de fangos aumenta hacia el tope y no presenta laminaciones de pumicita. La misma fue definida en función de la ausencia de las especies marinas y el aumento de la especie dominante Punctastriata glubokoensis (35-55\%) y las "fragilarioides pequeñas". La DZII corresponde al Holoceno tardío y se depositó con anterioridad a los $2.218 \pm 29$ años ${ }^{14} \mathrm{C}$ AP (2.140-2.309 años cal AP).

La totalidad de la sucesión sedimentaria LI estuvo dominada por diatomeas pennadas arrafídeas pequeñas de la familia Fragilariaceae. La especie dominante fue Punctastriata glubokoensis alcanzando abundancias máximas de 55\% (Figuras 2 y $3 \mathrm{~A}-\mathrm{B}$ ). Como constituyentes de la flora acompañante se registró un conjunto estable de especies de la misma familia generalizadas como "fragilarioides pequeñas" con abundancias variables según la sección (5-23\%). El mismo estuvo compuesto por Punctastriata lancettula (Figuras 3C-D), Pseudostaurosiropsis geocollegarum (Figuras 3E-F), Pseudostaurosira cf. oliveraiana Grana, Morales, Maidana \& Ector (Figuras 3G-H), Pseudostaurosira brevistriata (Grunow) Williams \& Round (Figura 3I), Staurosira construens Ehrenberg (Figura 3J), y Staurosira venter (Figuras $3 \mathrm{~K}-\mathrm{L}$ ). También estuvieron representadas en la flora acompañante especies dulceacuícolas planctónicas como Stephanodiscus agassizensis (Figuras 30 y Q), Aulacoseira granulata, epífitas como Cocconeis placentula Ehrenberg y dos especies ticoplanctónicas marino-costeras, Paralia sulcata (Figuras 3M y P) y Cymatosira belgica (Figura 3N). 

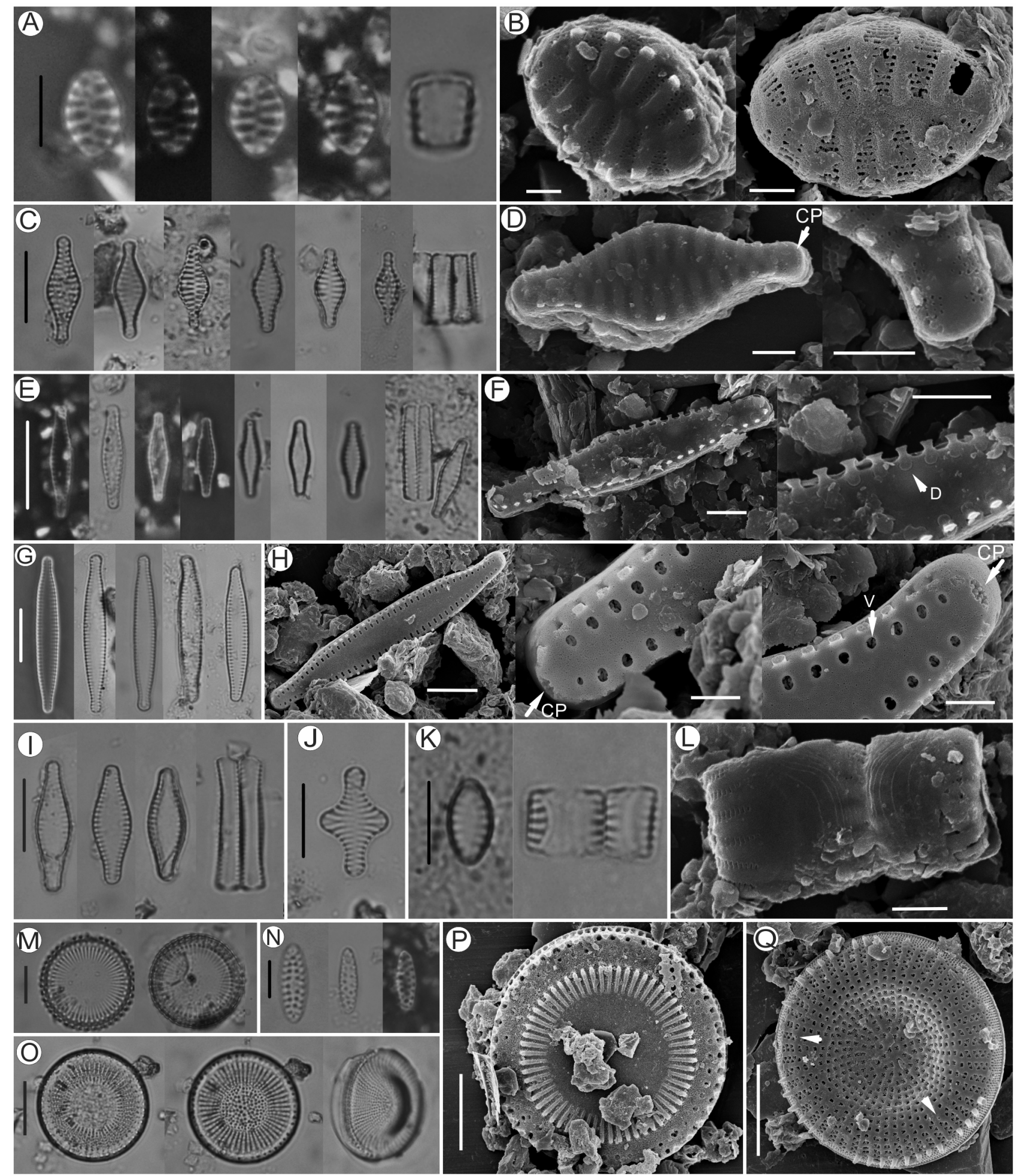

Figura 3. Fotomicrografías LM y MEB de diatomeas holocenas del delta del río Colorado. A-L, taxones de la familia Fragilareaceae: A-B, Punctastriata glubokoensis; C-D, Punctastriata lancettula; E-F, Pseudostaurosiropsis geocollegarum; G-H, Pseudostaurosira cf. oliveraiana; I, Pseudostaurosira brevistriata; J, Staurosira construens; K-L, Staurosira venter; $\mathbf{M}, \mathbf{P}$, Paralia sulcata; $\mathbf{N}$, Cymatosira belgica; $\mathbf{O}, \mathbf{Q}$, Sthephanodiscus agassizensis. Abreviaturas: $\mathbf{C P}$, campo de poros; $\mathbf{V}$, areola ocluida por volae; $\mathbf{D}$, proyección superficial en forma de disco que cubre la abertura externa de la areola. Escalas (LM): A y $\mathrm{N}=5 \mu \mathrm{m} ; \mathrm{B}-\mathrm{M}, \mathrm{O}-\mathrm{Q}=10 \mu \mathrm{m}$. Escalas (MEB): $\mathrm{B}=1 \mu \mathrm{m} ; \mathrm{D}, \mathrm{F}, \mathrm{L}=2 \mu \mathrm{m} ; \mathrm{H}, \mathrm{P}, \mathrm{Q}=5 \mu \mathrm{m}$ (H detalle de ápices, escala $=1 \mu \mathrm{m})$.

Figure 3. LM and MEB photomicrographs of holocene diatoms of the Colorado River Delta. A-L, taxa of the family Fragilareaceae; A-B, Punctastriata glubokoensis; C-D, Punctastriata lancettula; E-F, Pseudostaurosiropsis geocollegarum; G-H, Pseudostaurosira cf. oliveraiana; I, Pseudostaurosira brevistriata; J, Staurosira construens; K-L, Staurosira venter; M, P, Paralia sulcata; N, Cymatosira belgica; $\mathbf{O}$, Q, Sthephanodiscus agassizensis. Abbreviations: CP, pore field; $\mathbf{V}$, areola occluded by volae; $\mathbf{D}$, disc-shaped surface projection covering the outer opening of the areola. Scale bars (LM): A and $\mathrm{N}=5 \mu \mathrm{m}$; B-M, O-Q $=10 \mu \mathrm{m}$. Scale bars (MEB): $\mathrm{B}=1 \mu \mathrm{m} ; \mathrm{D}, \mathrm{F}, \mathrm{L}=2 \mu \mathrm{m} ; \mathrm{H}, \mathrm{P}, \mathrm{Q}=5 \mu \mathrm{m}(\mathrm{H}$ detail, scale $=1 \mu \mathrm{m})$. 


\section{DISCUSIÓN}

\section{Fragilarioides fósiles del río Colorado}

La sucesión sedimentaria holocena LI en el río Colorado está dominada por representantes pequeños de la familia Fragilariaceae, siendo Punctastriata glubokoensis la especie más abundante. Los requerimientos ecológicos de las "fragilarioides pequeñas" no son bien conocidos en la bibliografía. Algunos estudios al respecto señalan que son ubicuas en ambientes someros con escasa penetración de luz (Gell et al., 2007), lo cual es consistente con el ambiente sedimentario deltaico de río Colorado que posee una importante carga sedimentaria psamítica fina (Cappannini $\&$ Lores, 1966). Estos taxones son abundantes en ambientes litorales lénticos y lóticos, donde sus cadenas se asocian a los sedimentos finos, arenas, rocas, algas o macrófitas sumergidas (Morales et al., 2010; Cejudo-Figueiras et al., 2011; García et al., 2017). Se consideran especies planctónicas facultativas o ticoplanctónicas (Gell et al., 2007) y poseen tolerancia a altas concentraciones de nutrientes (Schmidt et al., 2004).

De forma consistente con los resultados obtenidos en LI, la dominancia de diatomeas con hábito ticoplanctónico se ha registrado en otros deltas como el del río Ebro (España). La misma fue interpretada por Benito et al. (2015) como indicios de hábitats con una columna de agua poco desarrollada y condiciones ambientales con un cierto grado de inestabilidad física (escasa disponibilidad luz e importante turbulencia por vientos y mareas). Sin embargo, la dominancia de especies ticoplanctónicas también puede responder a la dinámica sedimentaria y no sólo a la turbulencia. Una unión parcial al sustrato y la resuspensión continua en la columna de agua, podría evitar el enterramiento de aquellas diatomeas bentónicas que no poseen la capacidad de desplazarse (nomóviles), como es el caso de las arrafídeas, permitiendo su desarrollo en ambientes con importante carga sedimentaria (Underwood \& Paterson, 1993).

Respecto a los géneros hallados, los registros de Punctastriata y Pseudostaurosiropsis en general son muy escasos en la bibliografía y en particular en América del Sur. Los especímenes del género Punctastriata poseen estrías multiseriadas con areolas ocluidas internamente con volae, lo cual sólo puede resolverse mediante MEB (Figura 3B). Las especies de este género se diferencian según la morfología de la valva, la presencia de campos de poros y la forma de las espinas (Morales, 2005). Punctastriata glubokoensis es una especie elíptica heteropolar robusta pequeña con rangos de tamaño que varían entre 5,5 y $7 \mu \mathrm{m}$ (Figura 3A). Fue descripta originalmente en el perifiton marginal del lago Glubokoe en Rusia (Williams et al., 2009). P. glubokoensis suele ser usualmente confundida mediante microscopía óptica con otras especies como Staurosira venter o Staurosirella pinnata (Paull et al., 2008; Morales et al., 2013; Vélez-Agudelo et al., 2017). Punctastriata lancettula es la segunda especie dentro del género hallada en la secuencia LI con abundancias de 5 a $10 \%$. Posee valvas lanceoladas a cruciformes, heteropolares en especímenes elongados y un campo de poros pequeño o subdesarrollado en uno de los ápices (Hamilton
\& Siver, 2008; Figura 3C). Ambas especies se registraron recientemente en Argentina, en muestras de sedimentos superficiales del río Colorado (Vélez-Agudelo et al., 2017). Según estos autores, $P$. glubokoensis presenta una tolerancia a la salinidad de 0 a $2,1 \%$, mientras que $P$. lancettula tolera rangos más restringidos ( 0 a 1,68\%; Vélez-Agudelo et al., 2017). En Curitiba, Paraná (Brasil) se identificó una especie morfológicamente similar a $P$. lancettula que posee ambos ápices con campos de poros: Punctastriata mimetica, la cual se encontró adherida a macrófitas sumergidas en el embalse Passaúna, moderadamente eutrofizado (Bertolli et al., 2010).

Pseudostaurosiropsis geocollegarum es un taxón de pequeño tamaño que requiere de MEB para ser identificado a nivel de género y especie. El frústulo es isovalvar, las valvas son levementes lanceoladas a lineares y angostas (Figuras 3E y F), y presentan campos de poros reducidos o ausentes. Sus estrías son marginales y están compuestas por dos o múltiples areolas elongadas transapicalmente sin oclusiones internas ( $\sin$ volae). Posee como carácter diagnóstico particular una proyección de la superficie valvar en forma de disco que cubre la abertura externa de la areola (Morales, 2001, 2002; Li et al., 2019; Figura 3F). Esta especie posee poblaciones discretas como parte del perifiton de sistemas lóticos de América del Norte (Connecticut y Wisconsin) y fue descripta en arroyos y canales de Florida, estando presente en aguas ligeramente alcalinas ( $\mathrm{pH} 7,1-8,3)$, con conductividades altas (458-1120 $\mu \mathrm{S} / \mathrm{cm}$ ) y condiciones eutróficas (Morales, 2002). En Florida, se identificó una variedad tri-radiada (Morales, 2005). $P$. geocollegarum es abundante en sedimentos superficiales de lagunas costeras asociadas al delta del Ebro, con condiciones dulceacuícolas y conductividades promedio de $10 \mathrm{mS} / \mathrm{cm}$ (Benito et al., 2015) y es dominante en la base de una sucesión sedimentaria costera de Galápagos donde fue inferido un paleoambiente de laguna salobre sin influencia marina durante el Holoceno tardío (Seddon et al., 2011).

Las especies pertenecientes a los géneros Staurosira y Pseudostaurosira poseen mayor número de registros en la bibliografía, si bien han sufrido múltiples revisiones desde su descripción original (Cejudo-Figueiras et al., 2011; Grana et al., 2018). Staurosira es un género antiguo redefinido luego de la revisión del género Fragilaria. Posee estrías uniseriadas con areolas relativamente lineares y elongadas apicalmente. Las estrías cubren gran parte de la valva por lo que el área axial es estrecha. Sus espinas son huecas, marginales y se ubican en la interestría (Figuras $3 \mathrm{~K}$ y L). Además, presenta campos de poros poco desarrollados y bandas conectivales abiertas (Cejudo-Figueiras et al., 2011). Staurosira venter tolera rangos de salinidades de 1,2-1,4 (Nodine \& Gaiser, 2014), mientras que otros autores registran su ocurrencia en rangos más amplios: 1,4-18 (van Dam et al., 1994). Por su parte, el género Pseudostaurosira Williams \& Round (1987) continúa siendo un taxón problemático para la identificación mediante LM y MEB (Li et al., 2019). Posee estrías uniseriadas cortas formadas por escasas areolas redondeadas o elípticas transapicalmente, con importante desarrollo de volae. La disposición marginal de las estrías genera un área axial amplia en la valva (Figura $3 \mathrm{H}$ ). Posee campos de poros reducidos y 
por lo general las espinas interrumpen las estrías (Grana et al., 2018). Pseudostaurosira ha sido definido tradicionalmente como un género con representantes salobres-dulceacuícolas oligotróficos a mesotróficos [e.g. Pseudostaurosira brevistriata, Pseudostaurosira binodis (Ehrenberg) y Pseudostaurosira pseudoconstruens (Marciniak)]. Sin embargo, también posee especies abundantes en ambientes de mayor tenor salino (Li et al., 2019). Hassan et al. (2009) reconocen tolerancias de Staurosira venter de 1,28-18 \%o, mientras que Pseudostaurosira brevistriata tolera rangos de salinidad entre 1,05 y 14,5\%; ambas se hallan en el estuario de los ríos Quequén Grande y Quequén Salado y en la laguna de Mar Chiquita de Argentina.

Ambos géneros Staurosira y Pseudostaurosira han sido reconocidos ampliamente en sedimentos holocenos de Argentina, conformando ensambles salobre-dulceacuícolas en sistemas estuáricos y lacustres (Espinosa, 1994, 2008; Hassan et al., 2009, 2011; Espinosa et al., 2012; Prieto et al., 2014; Fayó et al., 2018; Guerrero et al., 2019, entre otros). Pseudostaurosira oliveraiana Grana, E. Morales, Maidana \& Ector fue descripta recientemente en sedimentos holocenos de lagos salinos del noroeste de Argentina (Grana et al., 2018); el mismo año esta especie fue registrada como Psudostaurosira sp. en los sedimentos holocenos del río Colorado (Fayó et al., 2018). Su presencia y persistencia en los ensambles de la planicie deltaica se confirma con estos nuevos resultados.

Actualmente el brazo principal que recorre el sitio de las Isletas conforma un canal fluvial salobre-dulceacuícola sin influencia marina. Los ensambles diatomológicos actuales contenidos en sus sedimentos superficiales están dominados por "fragilarioides pequeñas", que viven a salinidades de $0,5-0,9$, con una turbulencia moderada y un sustrato arenolimoso (Vélez-Agudelo et al., 2017). Estos ensambles se encuentran dominados por Staurosira venter y las especies descriptas anteriormente constituyen la flora acompañante. A la vez, los mismos taxones fragilarioides pequeños fueron registrados en un testigo sedimentario de la llanura deltaica del río Colorado a los $c a .4 .300$ años AP, donde fue inferido un paleoambiente deltaico semilótico salobre-dulceacuícola compatible con una laguna en medialuna (Fayó et al., 2018). Este nuevo registro expone la ubicuidad de las fragilarioides pequeñas en el ambiente fluvio-deltaico y amplía el rango temporal de su presencia en la región norte de Patagonia desde hace $c a$. 7.300 años AP hasta la actualidad.

\section{Reconstrucción paleoambiental del sitio LI}

A partir del análisis diatomológico de la sucesión sedimentaria LI y la integración de los resultados cronológicos y litológicos, se han podido reconstruir dos fases evolutivas desde el Holoceno medio hasta la actualidad. Las mismas se corresponden con DZI y DZII. Como se discutió en la sección anterior, la sucesión sedimentaria LI está dominada por las especies pennadas arrafídeas pequeñas salobre-dulceacuícolas de la familia Fragilariaceae. La abundancia y persistencia de esta asociación hasta la actualidad, indica que las condiciones fluviales salobre-dulceacuícolas se habrían establecido y dominado en el sitio de estudio durante los últimos 7.315 \pm 64 años ${ }^{14} \mathrm{C}$ AP (8.001-8.177 años cal AP). Sin embargo, en la sección basal de la secuencia (DZI) se identificaron también las especies marino-costeras Paralia sulcata y Cymatosira belgica (Figuras 2, 3M y N). En consecuencia, las fases evolutivas fueron definidas en función de la presencia y ausencia de la flora acompañante marino-costera, y los resultados se interpretaron en un contexto de fluctuaciones holocenas del nivel del mar. Debido a la sensibilidad de las diatomeas a los cambios en la salinidad, este grupo es uno de los proxies más utilizados en la interpretación de la evolución deltaica en función de las variaciones eustáticas (Castro et al., 2013).

La costa atlántica sudamericana ha sido extensamente afectada por las fluctuaciones del nivel del mar que siguieron al Último Máximo Glacial. Durante el período entre $c a$. 18.000 y 7.000 años AP, el mejoramiento climático y la deglaciación, produjeron el incremento eustático del nivel del mar (transgresión postglaciaria). Inicialmente el aumento relativo ocurrió de forma acelerada lo cual produjo un ingreso del agua marina hacia el continente (Cavallotto et al., 2004; Violante \& Parker, 2004; Schnack et al., 2005). La tendencia transgresiva iniciada durante el Pleistoceno tardío continuó hasta el Holoceno medio, sobrepasando el nivel marino actual $c a$. de los 7000 años AP y alcanzó una cota máxima ca. 6.000-4.000 años AP (Isla, 1989; Cavalotto et al., 2004). Con posterioridad al máximo transgresivo holoceno, el nivel relativo del mar disminuyó y continúa haciéndolo en el hemisferio sur, de manera que la fase regresiva resultante dio lugar a las morfologías costeras actuales (Isla, 2013). Se han propuesto distintas curvas de cambios relativos del nivel del mar en diferentes regiones costeras de Argentina (Isla, 1989; Aguirre \& Whatley, 1995; Gómez \& Perillo, 1995; Isla \& Espinosa, 1998; Cavallotto et al., 2004; Spagnuolo, 2005; Isla, 2013; Prieto et al., 2017). Estas curvas coinciden en la tendencia general de los cambios, sin embargo, poseen particularidades en respuesta a la variabilidad regional y local como por ejemplo la cota máxima del nivel del mar (Schnack et al., 2005). Las reconstrucciones respecto a los cambios del nivel del mar en la costa argentina son coincidentes con las tendencias documentadas para las costas de Brasil (Martin et al., 2003; Angulo et al., 2006), Uruguay (Bracco et al., 2011; Martínez \& Rojas, 2013), la costa atlántica de Sud África (Compton, 2001) y la costa este de Australia (Nakada \& Lambeck, 1989; Sloss et al., 2007).

En este contexto, la coocurrencia de especies con diferentes tolerancias salinas en la DZI de LI, puede explicarse por la mezcla de especies autóctonas y alóctonas. Debido a la persistencia y alta abundancia de las especies "fragilarioides pequeñas", esta asociación salobre-dulceacuícola se considera autóctona; las especies marinas, por el contrario, presentaron baja abundancia y un estado de preservación pobre, lo cual fue interpretado como indicios de transporte. Según los antecedentes bibliográficos el crecimiento óptimo de la especie Paralia sulcata se restringe a ambientes marino/salobres con límites mínimos de salinidad de 10\%o (Zong, 1997), por lo que un paleoambiente salobre-dulceacuícola habría sido poco favorable para su crecimiento. La incompatibilidad ecológica 
de las especies costero-marinas en un entorno fluvial y un estado de preservación pobre permite inferir que Paralia sulcata y Cymatosira belgica son especies alóctonas que fueron transportadas río arriba desde el ambiente marinocostero durante el Holoceno medio.

El máximo porcentaje de especies marino-costeras alóctonas de LI ocurrió a los $7.315 \pm 64$ años ${ }^{14} \mathrm{C}$ AP (8.0018.177 años cal AP), lo cual se correlaciona temporalmente con niveles del mar más alto que el actual. Estas evidencias permiten inferir que la acción de las mareas alcanzó el sitio LI e influyó en la composición de los ensambles de diatomeas, actuando principalmente como agente de transporte de especies marinas río arriba. Durante este período además se identifican niveles de pumicitas redondeadas de granulometrías arenosas medias, las cuales pudieron ser acumuladas en niveles máximos de agua asociados a la acción mareal del entorno mesomareal.

De acuerdo con la evidencia malacológica, durante la transgresión holocena la línea de costa en la planicie deltaica de río Colorado habría sobrepasado la línea de costa actual hacia el Oeste y se habría ubicado a $16 \mathrm{~km}$ río arriba $c a$. 6.600 años ${ }^{14} \mathrm{C} \mathrm{AP}$ (Codignotto \& Weiler, 1980). El transporte alóctono de especies marinas hacia el sitio LI, da indicios de una posición de la línea de costa cercana al sitio LI durante la transgresión del Holoceno, lo cual es consistente con la posición de los cordones litorales en la planicie deltaica (Weiler, 1980). Al igual que se infiere en la base de la sucesión sedimentaria LI, una máxima influencia marina con anterioridad a los $c a$. 7.000 años ${ }^{14} \mathrm{C} \mathrm{AP}$, fue registrada en otras sucesiones estuáricas holocenas como las de río Negro (Escandell et al., 2009), Mar Chiquita (Hassan et al., 2011) y río Quequén Grande (Espinosa et al., 2012).

Con posterioridad a los $c a .7 .300$ años AP el porcentaje de especies marino-costeras alóctonas en LI disminuye gradualmente hasta desaparecer a los $c a$. 2.200 años AP. En el estuario del río Hudson, la caída en la abundancia de P. sulcata fue asociada a la disminución de la salinidad (Weiss et al., 1978), mientras que en la Bahía de Chesapeake se atribuyó a un mayor aporte de agua dulce y al aumento de la sedimentación (Cooper, 1995). La reducción gradual en la abundancia de P. sulcata y otras especies marino-costeras alóctonas en DZII entre los $7.315 \pm 64$ y $2.218 \pm 29$ años ${ }^{14} \mathrm{C}$ AP(DZIB), puede asociarse entonces a una disminución en la influencia marina durante la etapa regresiva, así como también a un incremento de la influencia fluvial debido al desarrollo del lóbulo deltaico y la progradación de la línea de costa. El establecimiento de las condiciones fluviales se registra durante el Holoceno tardío ( $c a .2 .218 \pm 37$ años ${ }^{14} \mathrm{CAP}$ ), en un contexto de costa regresiva, donde las condiciones salobresdulceacuícolas del sitio LI favorecieron el crecimiento de los taxones fragilarioides hasta la actualidad.

Durante la segunda fase evolutiva del sitio (ca. 2.200 años AP y hasta la actualidad - DZII), se establecieron las condiciones fluviales predominantes, similares a las que actualmente caracterizan el brazo principal del río Colorado. Las mismas habrían favorecido el crecimiento óptimo de especies fragilarioides pequeñas salobre-dulceacuícolas asociadas al sustrato areno-limoso y a la vegetacion marginal submergida y a la vez, habrían permitido el desarrollo de especies planctónicas acompañantes tolerantes a la turbidez como Stephanodiscus agassizensis y Aulacoseira granulata en la columna de agua.

\section{CONCLUSIONES}

La dominancia y persistencia de las diatomeas fragilarioides pequeñas en los ensambles fósiles del testigo LI, indican que las condiciones fluviales salobre-dulceacuícolas en el sitio se habrían establecido y dominado durante los últimos $c a$. $7.315 \pm 64$ años ${ }^{14} \mathrm{C}$ AP. Esta nueva evidencia micropaleontológica constituye el registro más temprano de los géneros Pseudostaurosiropsis y Punctastriata en Argentina, y permite visualizar la ubicuidad de las fragilarioides pequeñas como componente estable en los ensambles fluvio-deltaicos del río Colorado. Además, la presencia de taxones marinocosteros alóctonos en la base del testigo, dan indicios de que el sitio LI (ubicado a $22 \mathrm{~km}$ de la costa actual), fue afectado por las fluctuaciones holocenas del nivel del mar. Durante la transgresión del Holoceno medio, la mayor influencia marina y una linea de costa ubicada $16 \mathrm{~km}$ tierra adentro (respecto a la actual), habrían favorecido el transporte de especies costero-marinas río arriba hasta el sitio. Mediante el análisis micropaleontológico y sedimentológico pudo inferirse que la secuencia sedimentaria LI representa la colmatación de un canal fluvial-estuarino con influencia mareal a los $c a .7 .000$ años AP que evoluciona gradualmente hacia un canal fluvial salobre-dulceacuícola similar al río actual a los $c a .2 .200$ años ${ }^{14} \mathrm{C}$ AP. El establecimiento de las condiciones fluviales predominantes se asocia a la progradación del lóbulo deltaico hacia el Este durante los últimos ca. 7.000 años ${ }^{14} \mathrm{C}$ AP y a los avances y retrocesos costeros asociados a las variaciones eustáticas del Holoceno.

\section{AGRADECIMIENTOS}

Los autores deseamos agradecer al C.A. Vélez-Agudelo y al J. Bedmar por su colaboración en las tareas de campo y la extracción del testigo, y a M. Oppedisano por su asesoramiento y asistencia en el MEB. Este estudio fue financiado por la Agencia Nacional de Promoción Científica y Tecnológica (PICT 1146/16).

\section{REFERENCIAS}

Aguirre, M.L. \& Whatley, R.C. 1995. Late Quaternary marginal marine deposits and palaeoenvironments from northeastern Buenos Aires Province, Argentina: a review. Quaternary Science Reviews, 14:223-254. doi:10.1016/02773791(95)00009-E

Angulo, R.J.; Lessa, G.C. \& Souza, M.C. 2006. A critical review of mid-to late- Holocene sea-level fluctuations on the eastern Brazilian coastline. Quaternary Science Reviews, 25:486-506. doi:10.1016/j.quascirev.2005.03.008 
Battarbee, R.W.; Jones, V.J.; Flower, R.J.; Cameron, N.G.; Bennion, H.; Carvalho, L. \& Juggins, S. 2001. Diatoms. In: J.P. Smol; H.J.B. Birks; W.M. Last; R.S. Bradley \& K. Alverson (eds.) Tracking environmental change using lake sediments: terrestrial, algal, and siliceous indicators, Springer, p. 155-202. doi:10.1007/0-306-47668-1 8

Benito, X.; Trobajo, R. \& Ibáñez, C. 2015. Benthic diatoms in a Mediterranean delta: ecological indicators and a conductivity transfer function for paleoenvironmental studies. Journal of Paleolimnology, 54:171-188. doi:10.1007/s10933-015-9845-3

Bennett, K.D. 1996. Determination of the number of zones in a biostratigraphical sequence. New Phytologist, 132:55-170. doi:10.1111/j.1469-8137.1996.tb04521.x

Bertolli, L.M.; Tremarin, P.I. \& Ludwig, T.A. 2010. Diatomáceas perifíticas em Polygonum hydropiperoides Michaux, reservatório do Passaúna, Região Metropolitana de Curitiba, Paraná, Brasil. Acta Botanica Brasilica, 24:1065-1081. doi:10.1590/S010233062010000400022

Biasotti, A.; Álvarez, B.; Bazán, I. \& Martinez de Fabricius, A.M. 2013. Biodiversidad y distribución temporal fitoplanctónica en río Colorado, La Pampa, Argentina. Biológicas, 15:38-51.

Biasotti, A.; Álvarez, S.; Bazán, G.I. \& Martínez de Fabricius, A. 2014. Variación estacional de la comunidad microfitoplanctónica del curso medio del río Colorado (La Pampa, Argentina). Biología Acuática, 30:249-258.

Bigler, C. \& Hall, R.I. 2002. Diatoms as indicators of climatic and limnological change in Swedish Lapland: a 100-lake calibration set and its validation for paleoecological reconstructions. Journal of Paleolimnology, 27:97-115. doi:10.1023/A:1013562325326

Blasi, A. 1986. Sedimentología del Río Colorado. Facultad de Ciencias Naturales y Museo, Universidad Nacional de La Plata, Tesis Doctoral, 238 p.

Bracco, R.; García-Rodríguez, F.; Inda, H.; Del Puerto, L.: Castiñeira, C. \& Panario, D. 2011. Niveles relativos del mar durante el Pleistoceno final-Holoceno en la costa de Uruguay. In: F.G. Rodríguez (ed.) Holoceno en la zona costera de Uruguay, Universidad de la República, p. 65-92.

Cappannini, D.A. \& Lores, R.R. 1966. Los suelos del valle inferior del río Colorado (Provincia de Buenos Aires). Buenos Aires, Instituto Nacional de Tecnología Agropecuaria, $236 \mathrm{p}$.

Castro, D.F.: Rossetti, D.F.; Cohen, M.C.; Pessenda, L.C. \& Lorente, F.L. 2013. The growth of the Doce River Delta in northeastern Brazil indicated by sedimentary facies and diatoms. Diatom Research, 28:455-466. doi:10.1080/0269249X.2013.841100

Cavallotto, J.L.; Violante, R.A. \& Parker, G. 2004. Sea-level fluctuations during the last 8600 years in the de la Plata River (Argentina). Quaternary International, 114:155-165. doi:10.1016/S1040-6182(03)00050-8

Cejudo-Figueiras, C.; Morales, E.A.; Wetzel, C.E.; Blanco, S.; Hoffmann, L. \& Ector, L. 2011. Analysis of the type of Fragilaria construens var. subsalina (Bacillariophyceae) and description of two morphologically related taxa from Europe and the United States. Phycologia, 50:67-77. doi:10.2216/09-40.1

Charó, M.P.; Fucks, E.E. \& Gordillo, S. 2015. Late PleistoceneRecent marine malacological assemblages of the Colorado River delta (south of Buenos Aires Province): paleoecology and paleoclimatology. Quaternary International, 377:52-70. doi:10.1016/j.quaint.2015.05.025

Codignotto, J. \& Weiler, N. 1980. Evolución Morfodinámica del sector costero comprendido entre punta Laberinto e isla Olga, provincia de Buenos Aires. In: SIMPOSIO SOBRE PROBLEMAS GEOLÓGICOS DEL LITORAL ATLÁNTICO
BONAERENSES. Resúmenes Mar del Plata, Comisión de Investigaciones Científicas de la Provincia de Buenos Aires, p. $35-43$.

COIRCO. 2018. Comité Interjurisdiccional del Río Colorado. Programas de calidad del medio acuático. Subprograma Calidad del Medio Acuático. Secretaría de Energía de la Nación, Grupo Interempresario. Available at http://www.coirco.gov.ar/centrode-documentacion/; accessed on 11/17/ 2018.

Compton, J.S. 2001. Holocene sea-level fluctuations inferred from the evolution of depositional environments of the southern Langebaan Lagoon salt marsh, South Africa. The Holocene, 11:395-405. doi:10.1191/095968301678302832

Cooper, S.R. 1995. Chesapeake Bay watershed historical land use: impact on water quality and diatom communities. Ecological Applications, 5:703-723. doi:10.2307/1941979

Cox, E.J. 2012. Ontogeny, homology, and terminology - wall morphogenesis as an aid to character recognition and character state definition for pennate diatom systematics. Journal of Phycology, 48:1-31. doi:10.1111/j.1529-8817.2011.01081.x

Crosta, X. \& Koç, N. 2007. Diatoms: From Micropaleontology to Isotope Geochemistry. In: C. Hillaire-Marcel \& A. De Vernal (eds.) Proxies in Late Cenozoic paleoceanography, Amsterdam, Elsevier, p. 327-358 (Developments in Marine Geology 1). doi:10.1016/S1572-5480(07)01013-5

Denys, L. 1991/1992. A checklist of the diatoms in the Holocene deposits of the western Belgian coastal plain with a survey of their apparent ecological requirements. Geological Survey of Belgium, 246:1-41.

Díaz Pardo, C.A.; Echazú, D.M. \& Maidana, N.I. 2008. Diatomeas continentales como indicadoras de cambios climáticos en Patagonia. In: A.V. Volpedo \& L. Fernández Reyes(eds.) Efecto de los cambios globales sobre la biodiversidad, CYTED, p. 233-246.

Echazú, D.M. 2012. Biodiversidad de diatomeas en humedales del sur de la Provincia de Santa Cruz, Argentina. Facultad de Ciencias Exactas y Naturales. Universidad de Buenos Aires, Tesis Doctoral, $353 \mathrm{p}$.

Escandell, A.; Espinosa, M.A. \& Isla, F.I. 2009. Diatomeas como indicadoras de variaciones de salinidad durante el Holoceno tardío en el río Negro, Patagonia Norte, Argentina. Ameghiniana, 46:461-468.

Espinosa, M.A. 1994. Diatom paleoecology of the Mar Chiquita lagoon delta, Argentina. Journal of Paleolimnology, 10:17-23. doi:10.1007/BF00683142

Espinosa, M.A. 2008. Diatoms from Patagonia and Tierra del Fuego. Developments in Quaternary Sciences, 11:383-392. doi:10.1016/S1571-0866(07)10019-1

Espinosa, MA.; Hassan, G.S. \& Isla, F.I. 2012. Diatom-inferred salinity changes in relation to Holocene sea-level fluctuations in estuarine environments of Argentina. Alcheringa, 36:373-386. doi:10.1080/03115518.2012.657501

Espinosa, M.A. \& Isla, F.I. 2015. Modern diatom assemblages in surface sediments from meso-macrotidal estuaries of Patagonia, Argentina. Pan-American Journal of Aquatic Sciences, 10:2943. doi:10.1007/s10933-005-6444-8

Fayó, R. \& Espinosa, M.A. 2014. Reconstrucción paleoambiental de la planicie costera de Mar Chiquita (Provincia de Buenos Aires, Argentina) durante el Holoceno, basada en diatomeas. Ameghiniana, 51:510-528. doi:10.5710/ AMGH.13.10.2014.2812

Fayó, R.; Espinosa, M.A.; Vélez-Agudelo, C.A.; Pan, J. \& Isla, F.I. 2018. Diatom based reconstruction of Holocene hydrological 
changes along the Colorado River floodplain (northern Patagonia, Argentina). Journal of Paleolimnology, 60:427-443. doi:10.1007/s10933-018-0031-2

Flower, R.J.; Jones, V.J. \& Round, F.E. 1996. The distribution and classification of a problematic Fragilaria virescens var. exigua Grun./Fragilaria exiguiformis (Grun.) Lange-Bertalot: a new species or a new genus? Diatom Research, 11:41-5. doi:10.10 80/0269249X.1996.9705363

Galea, M.; Bazán, G.; Álvarez, S. \& Martínez de Fabricius, A. 2014. Estudio del fitoplancton aguas arriba y aguas abajo del embalse Casa de Piedra, río Colorado (La Pampa, Argentina). Biología Acuática, 30:287-300.

García, M.L. \& Maidana, N.I. 2015. Diatomeas (Bacillariophyceae) subfósiles del Brazo Blest del Lago Nahuel Huapi, Argentina. Boletín de la Sociedad Argentina de Botánica, 50:123-140. doi:10.31055/1851.2372.v50.n2.11657

García, M.L.; Maidana, N.I.; Ector, L. \& Morales, EA. 2017. Staurosira patagonica sp. nov., a new diatom (Bacillariophyta) from southern Argentina, with a discusión on the genus Staurosira Ehrenberg. Nova Hedwigia, 146:103-123. doi:10.1127/14389134/2017/123

Garibotti, I.A.; Ferrario, M.E.; Almandoz, G.O. \& Castaños, C. 2011. Seasonal diatom cycle in Anegada Bay, El Rincón estuarine system, Argentina. Diatom Research, 26:227-241. doi:10.108 0/0269249X.2011.604158

Gell, P.; Tibby, J.; Little, F.; Baldwin, D. \& Hancock, G. 2007. The impact of regulation and salinisation on floodplain lakes: the lower River Murray, Australia. Hydrobiologia, 591:135-146. doi:10.1007/s10750-007-0806-3

Gómez, E. \& Perillo, G. 1995. Submarine outcrops underneath shoreface connected sand ridges, outer Bahía Blanca Estuary, Argentina. Quaternary of South America and Antarctica Peninsula, 9:23-37.

González Uriarte, M. 1984. Características geomorfológicas de la porción continental que rodea la Bahía Blanca, Provincia de Buenos Aires. In: CONGRESO GEOLÓGICO ARGENTINO, 9, 1984. Actas III, San Carlos de Bariloche, p. 556-576.

Grana, L.; Morales E.A.; Maidana, N.I. \& Ector, L. 2018. Two new species of Staurosira and Pseudostaurosira (Bacillariophyta) from the highlands of Argentina (south-central Andes) and two new nomenclatural combinations. Phytotaxa, 365:60-72. doi:10.11646/phytotaxa.365.1.2

Guerrero, J.M.; García, M.L. \& Morales, E.A. 2019. Staurosirella andino-patagonica sp. nov. (Bacillariophyta) from lake sediments in Patagonia, Argentina. Phytotaxa, 402:131-144. doi:10.11646/phytotaxa.402.3.1

Guiry, M.D. \& Guiry, G.G. 2020. Algaebase. World-wide electronic publication, National University of Ireland, Galway. Available at http://www.algaebase.org; accessed on 11/21/2019.

Hamilton, P.B. \& Siver, P.A. 2008. The type for Fragilaria lancettula Schumann 1867 and transfer to the genus Punctastriata as Punctastriata lancettula (Schum.) Hamilton \& Siver nov. comb. Diatom Research, 23:355-365. doi:10.1080/026924 9X.2008.9705762

Hassan, G.S.; Espinosa, M.A. \& Isla, F.I. 2009. Diatom-based inference model for paleosalinity reconstructions in estuaries along the northeastern coast of Argentina. Palaeogeography, Palaeoclimatology, Palaeoecology, 275:77-91. doi:10.1016/j. palaeo.2009.02.020

Hassan, G.S.; Espinosa, M.A. \& Isla, F.I. 2011. Fluctuaciones de salinidad durante el Holoceno en la laguna costera de Mar
Chiquita (Provincia de Buenos Aires): una aproximación cuantitativa basada en diatomeas. Ameghiniana, 48:496-507. doi:10.5710/AMGH.v48i4(326)

Hermany, G.; Souza, P.A. \& Torga, L.C. 2013. Paleoecologia do sistema Pinguela-Palmital-Malvas, Holoceno da Bacia de Pelotas, RS, Brasil: uma abordagem focada na utilização de análises multivariadas para obtenção de diatomáceas descritora. Pesquisas em Geociências, 40:31-49. doi:10.22456/18079806.40833

Isla, F.I. 1989. The Southern Hemisphere sea level fluctuation. Quaternary Science reviews, 8:359-368.

Isla, F.I. 2013. The flooding of the San Matías Gulf: the Northern Patagonia sea-level curve. Geomorphology, 203:60-65. doi:10.1016/j.geomorph.2013.02.013

Isla, F.I. \& Espinosa, M. 1998. Modelo sedimentario de colmatación de pequeños estuarios dominados por limo, provincia de Buenos Aires. In: REUNIÓN ARGENTINA DE SEDIMENTOLOGÍA, 7, 1998. Actas, Buenos Aires, p. 24-36.

Juggins, S. 2015. Rioja: analysis of Quaternary science data. R package version (0.95). Available at http://cran.r-project.org/ package $=$ rioja; accessed on 07/15/2020.

Kociolek, J.; Balasubramanian, K.; Blanco, S.; Coste, M.; Ector, L.; Liu, Y.; Kulikovskiy, M.; Lundholm, N.; Ludwig, T.; Potapova, M.; Rimet, F.; Sabbe, K.; Sala, S.; Sar, E.; Taylor, J.; Van de Vijver, B.; Wetzel, C.E.; Williams, D.M.; Witkowski, A. \& Witkowski, J. 2018. DiatomBase. Available at http://www. diatombase.org; accessed on 10/2019.

Li, C.L.; Ashworth, M.P.; Witkowski, A.; Lobban, C.S.; Zgłobicka, I.; Kurzydłowski, K.J. \& Qin, S. 2016. Ultrastructural and molecular characterization of diversity among small araphid diatoms all lacking rimoportulae. I. Five new genera, eight new species. Journal of Phycology, 52:1018-1036. doi:10.1111/ jpy. 12460

Li, C.L; Witkowski, A.; Ashworth, M.P.; Dąbek, P.; Sato, S.; Zgłobicka, I.; Witak, M.; Khim, J.S. \& Kwon, C.J. 2019. The morphology and molecular phylogenetics of some marine diatom taxa within the Fragilariaceae, including twenty undescribed species and their relationship to Nanofrustulum, Opephora and Pseudostaurosira. Phytotaxa, 355:1-104. doi:10.11646/ phytotaxa.355.1.1

Maidana, N.I. \& Díaz Villanueva, V. 2001. Diatomeas de lagos oligotróficos andinos (Provincia de Neuquén, Argentina). Boletín de la Sociedad Argentina de Botánica, 36:15-27.

Martin, L.; Dominguez, J.M. \& Bittencourt, A.C. 2003. Fluctuating Holocene sea levels in eastern and southeastern Brazil: evidence from multiple fossil and geometric indicators. Journal of Coastal Research, 19:101-124. doi:10.2307/4299151

Martínez, S. \& Rojas, A. 2013. Relative sea level during the Holocene in Uruguay. Palaeogeography, Palaeoclimatology, Palaeoecology, 374:123-131. doi:10.1016/j.palaeo.2013.01.010

McCormac, F.G.; Hogg, A.G.; Blackwell, P.G.; Buck, C.E.; Higham, T.F. \& Reimer, P.J. 2004. SHCal04 Southern Hemisphere calibration, 0-11.0 cal kyr BP. Radiocarbon, 46:1087-1092. doi:10.1017/S0033822200033014

Medlin, L.; Yang, I. \& Sato, S. 2012. Evolution of the diatoms. VII. Four gene phylogeny assessed the validity of selected araphid genera. Nova Hedwigia, 141:505-514.

Melo, W.; Perillo, G.; Perillo, M.; Schilizzi, R. \& Piccolo, M.C. 2013. Late Pleistocene Holocene deltas in southern Buenos Aires Province, Argentina. IAHS-AISH Proceedings and Reports, 358:187-195. 
Metzeltin, D. \& Lange-Bertalot, H. 2007. Tropical diatoms of South America II. Special remarks on biogeographic disjunction. Iconographia Diatomológica, 18:1-877.

Morales, E.A. 2001. Morphological studies in selected fragilarioid diatoms (Bacillariophyceae) from Connecticut waters (USA). Proceedings of the Academy of Natural Sciences of Philadelphia, 151:105-120. doi:10.1635/0097-3157(2001)151[0105:MSIS FD]2.0.CO;2

Morales, EA. 2002. Studies in selected fragilarioid diatoms of potential indicator value from Florida (USA) with notes on the genus Opephora Petit (Bacillariophyceae). Limnologica, 32:102-113. doi:10.1016/S0075-9511(02)80002-0

Morales, E.A. 2005. Observations of the morphology of some known and new fragilarioid diatoms (Bacillariophyceae) from rivers in the USA. Phycological Research, 53:113-133. doi:10.1111/ j.1440-183.2005.00378.x

Morales, E.A.; Guerrero, J.M.; Wetzel, C.E.; Sala, S. \& Ector, L. 2013. Unraveling the identity of Fragilaria pinnata Ehrenberg and Staurosira pinnata Ehrenberg: research in progress on a convoluted story. Cryptogamie, Algologie, 34:89-102. doi:10.7872/crya.v34.iss2.2013.89

Morales, E.A.; Wetzel, C.E. \& Ector, L. 2010. Two short-striated species of Staurosirella (Bacillariophyceae) from Indonesia and the United States. Polish Botanical Journal, 55:107-117.

Morales, E.A.; Wetzel, C.E.; Rivera, S.F.; Van de Vijver, B. \& Ector, L. 2014. Current taxonomic studies on the diatom flora (Bacillariophyceae) of the Bolivian Altiplano, South America, with possible consequences for palaeoecological assessments. Journal of Micropalaeontology, 33:121-129. doi:10.1144/ jmpaleo2014-007

Nakada, M. \& Lambeck, K. 1989. Late Pleistocene and Holocene sea-level change in the Australian region and mantle rheology. Geophysical Journal International, 96:497-517. doi:10.1111/ j.1365-246X.1989.tb06010.x

Nodine, E.R. \& Gaiser, E.E. 2014. Distribution of diatoms along environmental gradients in the Charlotte harbor, Florida (USA), estuary and its watershed: implications for bioassessment of salinity and nutrient concentrations. Estuaries and Coasts, 37:864-879. doi:10.1007/s12237-013-9729-6

Oksanen, J.; Blanchet, F.; Kindt, R.; Legendre, P.; Minchin, P.; O’Hara R.B; Solymos, P.; Stevens, M.H.H \& Wagner, H. 2015. vegan: community ecology package. Available at https://cran. rproject.org/package=vegan; accessed on 11/2018.

Paull, T.M.; Hamilton, P.B.; Gajewski, K. \& Le Blanc, M. 2008. Numerical analysis of small Arctic diatoms (Bacillariophyceae) representing the Staurosira and Staurosirella species complexes. Phycologia, 47:213-224. doi:10.2216/07-17.1

Prieto, A.R.; Mourelle, D.; Peltier, W.R.; Drummond, R.; Vilanova, I. \& Ricci, L. 2017. Relative sea-level changes during the Holocene in the Río de la Plata, Argentina and Uruguay: a review. Quaternary International, 442:35-49. doi:10.1016/j. quaint.2016.02.044

Prieto, A.R.; Romero, M.V.; Vilanova, I.; Bettis, E.A.; Espinosa, M.A.; Haj, A.E.; Gómez, L. \& Bruno, L. 2014. A multi-proxy study of Holocene environmental change recorded in alluvial deposits along the southern coast of the Pampa region, Argentina. Journal of Quaternary Science, 29:329-342. doi:10.1002/ jqs. 2703

R Development Core Team. 2015. R: language and environmental for statistical computing. Vienna. R Foundation for Statistical Computing.
Round, F.E.; Crawford, RM. \& Mann, D.G. 1990. Diatoms: biology and morphology of the genera. New York, Cambridge University Press, $747 \mathrm{p}$.

Schmidt, R.; Kamenik, C.; Lange-Bertalot, H. \& Rolf, K. 2004. Fragilaria and Staurosira (Bacillariophyceae) from sediment surfaces of 40 lakes in the Austrian Alps in relation to environmental variables, and their potential for palaeoclimatology. Journal of Limnology, 63:171-189. doi:10.4081/jlimnol.2004.171

Schnack, E.; Isla, F.I.; De Francesco, F. \& Fucks, E. 2005. Estratigrafía del Cuaternario marino tardío en la provincia de Buenos Aires. Geología y Recursos Minerales de la Provincia de Buenos Aires. In: CONGRESO GEOLÓGICO ARGENTINO, 16, 1995. Relatorio X, La Plata, p. 159-182.

Seddon, A.W.; Froyd, C.A.; Leng, M.J.; Milne, G.A. \& Willis, K.J. 2011. Ecosystem resilience and threshold response in the Galápagos coastal zone. Ecological Thresholds in Galápagos Mangroves, 6:e22376. doi:10.1371/journal.pone.0022376

Sloss, C.R.; Murray-Wallace, C.V. \& Jones, B.G. 2007. Holocene sea-level change on the southeast coast of Australia: a review. The Holocene, 17:999-1014. doi:10.1177/0959683607082415

Spagnuolo, J. 2005. Evolución geológica de la región costera-marina de Punta Alta, provincia de Buenos Aires. Universidad Nacional del Sur, Tesis Doctoral, 269 p.

Spalletti, L.A. \& Isla, F.I. 2003. Caracteristicas y evolucion del delta del Rio Colorado "Colú-leuvú", Provincia de Buenos Aires, República Argentina. Revista de la Asociación Argentina de Sedimentología, 10:23-37.

Sterrenburg, F.A.S. 2006. Cleaning diatom samples. Available at http://www.microscopy-uk.org.uk/mag/artaug06/fs-diatoms. html; accessed on 07/15/2020.

Stuiver, M.; Reimer, P. \& Reimer, R. 2005. CALIB 5.0.1 Computer program and documentation. Available at http://calib.org; accessed on $07 / 15 / 2020$.

Theriot, E.C.; Ashworth, M.P.; Nakov, T.; Ruck, E. \& Jansen, R.K. 2015. Dissecting signal and noise in diatom chloroplast protein encoding genes with phylogenetic information profiling. Molecular Phylogenetics and Evolution, 89:28-36. doi:10.1016/j.ympev.2015.03.012

Underwood, G.J. \& Paterson, D.M. 1993. Seasonal changes in diatom biomass, sediment stability and biogenic stabilization in the Severn Estuary. Journal of the Marine Biological Association of the United Kingdom, 73:871-887. doi:10.1017/ S0025315400034780

van Dam, H.; Mertens, A. \& Sinkeldam, J. 1994. A coded checklist and ecological indicator values of freshwater diatoms from the Netherlands. Netherland Journal of Aquatic Ecology, 28:117133. doi:10.1007/BF02334251

Vélez-Agudelo, C.A.; Espinosa, M.A; Fayó, R. \& Isla, F.I. 2017. Modern diatoms from a temperate river in South America: the Colorado River (North Patagonia, Argentina). Diatom Research, 32:133-152. doi:10.1080/0269249X.2017.1321046

Violante, R.A. \& Parker, G. 2004. The post-last glacial maximum transgression in the de la Plata River and adjacent inner continental shelf, Argentina. Quaternary International, 114:167181. doi:10.1016/S1040-6182(03)00036-3

Vos, P.C. \& de Wolf, H. 1993. Diatoms as a tool for reconstructing sedimentary environments in coastal wetlands; methodological aspects. Hydrobiologia, 269:285-296. doi:10.1007/BF00028027

Vouilloud A. \& Leonardi P. 2001. Bacillariophyceae de los canales de Corfo (Río Colorado, Buenos Aires, Argentina). Boletin de la Sociedad Argentina de Botánica, 36:229-241. 
Weiler, N. 1980. Evolución costanera en el área del delta del río Colorado. Revista de la Asociación Geológica Argentina, 35:440-442.

Weiss, D.; Geitzenauer, K. \& Shaw, F.C. 1978. Foraminifera, diatom and bivalve distribution in recent sediments of the Hudson estuary. Estuarine, Coastal and Shelf Science, 7:393-400. doi:10.1016/0302-3524(78)90091-9

Williams, D.M.; Chudaev, D.A. \& Gololobova, M.A. 2009. Punctastriata glubokoensis spec. nov., a new species of 'fragilarioid' diatom from Lake Glubokoe, Russia. Diatom Research, 24:479-48. doi:10.1080/0269249X.2009.9705814

Williams, D.M. \& Round, F.E. 1987. Revision of the genus Fragilaria. Diatom Research, 2:267-288. doi:10.1080/02692 49X.1987.9705004
Witkowski, A.; Lange-Bertalot, H. \& Metzeltin, D. 2000. Diatom Flora of Marine Coasts I. Iconographia Diatomologica, 7:1-925.

Zong, Y. 1997. Mid-and late-Holocene sea-level changes in Roudsea Marsh, northwest England: a diatom biostratigraphical investigation. The Holocene, 7:311-323. doi:10.1177/095968369700700307

Received in 29 December, 2019; accepted in 29 June, 2020. 